'Presidente de ALANAM y de la Academia Chilena de Medicina. ${ }^{2}$ Secretario Alterno de ALANAM y Secretario Académico de la Academia Chilena de Medicina.

\title{
Declaración Pública de la ALANAM sobre Políticas de Salud para Latinoamérica
}

\author{
ALEJANDRO GOIC G. ${ }^{1}$, RODOLFO ARMAS M. ${ }^{2}$
}

\section{The ALANAM statement on public health policy}

\begin{abstract}
The ALANAM (Association of Latin American National Academies of Medicine) statement on public health policy, issued following its 19th Congress, held October 28-30, 2010, in Santiago, Chile, declares that cardiovascular diseases, cancer, accidents and violence are the leading causes of death in the region, while in several of its member nations, emergent and re-emergent infectious diseases, malnutrition, and mother-child illnesses remain prevalent. The statement calls attention to the lack of functioning water supply and sewage systems in many villages and rural areas. After describing the social causes of the present state of public health in Latin America (poverty levels reaching upwards of $44 \%$ of the total population, or some 110 million people), it calls on governments, first, to spare no efforts in the task of eradicating extreme poverty in the short-term, and poverty in the long-term. Second, considering that about 15 million 3-to-6 year-olds have no access to education, it recommends extending educational services to these children, and to improve the quality of existing pre-school and primary education. Third, the statement calls for universal health care coverage and for equal access to good quality medical care for everyone, and for programs aimed at promoting healthy personal habits and self-care. In this regard, it also recommends that disease prevention programs be sustained over time, that national sanitary objectives be defined, and that its results be periodically reviewed. Fourth, it recommends that primary health care be extended to everyone, and that it be enhanced by improving coverage and coordination with secondary and tertiary level health care institutions. The statement lays special stress on the need for adopting public health policies aimed at lowering the cost of medicines; to this end, it calls for the creation of an official list of generic drugs. The statement ends by calling on governments to support public health research as a necessary step in tackling with greater efficiency the health problems still prevalent in the region.
\end{abstract}

(Rev Med Chile 2010; 138: 1558-1560).

Key words: Academies and Institutes; Health policy; Health transition; Public health.

L os Presidentes y Delegados Permanentes de las Academias Nacionales de Medicina de XIX Reunión de su Consejo Directivo en Santiago de Chile, del 28 al 30 de octubre de 2010 acuerdan, por unanimidad, emitir la siguiente Declaración sobre Políticas de Salud para la Región:

Considerando Que:
1. La salud es uno de los componentes de la calidad de vida y bienestar de las personas y está íntimamente ligada a los niveles de ingreso, de educación, al saneamiento ambiental, disponibilidad de agua potable y alcantarillado y otras condicionantes sociales. De allí que el progreso en salud requiere de crecimiento y desarrollo económicos, de políticas y estrategias sociales 
integrales, éticas y coherentes y de un uso eficiente de los recursos disponibles.

2. Los índices de pobreza en la Región son muy elevados, estimándose que un $44 \%$ de su población, unos 230 millones de personas, viven en la pobreza y, de ellos, alrededor de 110 millones en la pobreza extrema e indigencia. En esta realidad, un gran porcentaje de esa población no puede asumir los costos de la atención de salud, situación que los Estados deben considerar prioritariamente al organizar sus sistemas de atención médica y sanitaria. Por otra parte, la educación muestra deficiencias, especialmente en el nivel preescolar; se estima que 15 millones de niños entre 3 y 6 años de edad no están en el sistema escolar, lo que corresponde a un $40 \%$ de ese grupo etario.

3. Los Gobiernos de los Estados tienen el deber de velar por el derecho a la salud de todo ser humano, asegurando el acceso de toda la población a los servicios sanitarios y a la atención médica. Esto implica lograr una cobertura universal en salud y equidad en el acceso a este bien, a través de sistemas sanitarios que, adaptados a la realidad de cada país, consideren las necesidades de la comunidad al margen de la capacidad económica de las personas y de los intereses comerciales. La atención médica debe estar a cargo de médicos cuya idoneidad esté debidamente certificada y estén sujetos a las regulaciones para el ejercicio de la profesión, propias de cada país. Por su parte, las personas tienen el deber del autocuidado de la salud, adoptando hábitos de vida saludables que prevengan enfermedades que son no sólo una carga para ellas sino para toda la sociedad.

4. La situación de salud de la población latinoamericana es, en muchos aspectos, insatisfactoria, sin perjuicio de los avances que han logrado en esta materia que, entre otros aspectos, se refleja en la disminución de tasas de mortalidad y el aumento en la expectativa de vida, indicadores que, en todo caso, están aún lejos de los que exhiben los países desarrollados.

5. Actualmente, el perfil epidemiológico de los países latinoamericanos muestra, como principales causas de muerte, a las enfermedades cardiovasculares, el cáncer y los accidentes y violencia pero, en algunos de ellos, aún son muy importantes las enfermedades infecciosas emergentes y re-emergentes, el VIH/SIDA, el paludismo, las enfermedades prevalentes en la madre y el niño. También, en muchas comunidades hay carencias graves en la disponibilidad de agua potable y de alcantarillado.

6. El acceso de los pacientes a médicos generales y especialistas, el financiamiento de la atención, la calidad de la infraestructura sanitaria y la acogida que se brinda a los enfermos, son poco satisfactorios. En nuestros países se debe seguir ampliando el trabajo en la atención primaria de salud como estrategia eficiente y eficaz de atención de la población; sin embargo, ésta aún muestra deficiencias en su cobertura, funcionamiento en red y coordinación con los niveles más complejos.

7. Las acciones sistemáticas de promoción de la salud y prevención de enfermedades, indispensables para mejorar la salud colectiva, muestran debilidades e incoherencias y suelen carecer de continuidad en el tiempo.

8. El acceso de las personas a los medicamentos es limitado, debido al alto costo de ellos que, muchas veces, la mayoría de la población no está en condiciones de cubrir.

9. La investigación en medicina y salud, en términos comparativos, es incipiente, pese a ser indispensable para un adecuado conocimiento de la realidad médica y sanitaria de cada país, así como para priorizar los problemas de salud que es necesario enfrentar y resolver.

\section{Las Academias Nacionales de Medicina de Lati- noamérica, exhortan a los gobiernos de la región a:}

1. Hacer los mayores esfuerzos para actuar sobre las determinantes de la salud, definiendo objetivos e implementando estrategias para el crecimiento y desarrollo económico-social, que permitan eliminar, en el corto plazo, la pobreza extrema y la marginalidad y, en el mediano plazo, disminuir progresivamente la pobreza. Lograr en el plazo más breve cobertura universal en educación preescolar y primaria de 
calidad, mejorar la formación de los profesores e implementar tecnologías modernas de apoyo a la enseñanza. Así mismo, invoca a hacer efectivas políticas públicas adecuadas para lograr un medio ambiente saludable, impidiendo la instalación de industrias contaminantes, combatiendo la polución del aire, de los lagos, ríos y mares, extendiendo la forestación y las áreas verdes en las ciudades.

2. Definir objetivos sanitarios cuantificables y evaluables en plazos predeterminados, estableciendo metas e indicadores de salud. Asimismo, intensificar y extender las acciones de promoción de la salud y de prevención de las enfermedades transmisibles y no transmisibles, manteniéndolas en el tiempo y midiendo sus resultados.

3. Profundizar la estrategia de atención primaria de salud, extendiendo esta red asistencial a toda la población, haciéndola resolutiva y mejorando su coordinación con los niveles secundarios y terciarios. Por otra parte, exhorta a perfeccionar los servicios de atención de urgencias médicas y quirúrgicas, de modo que den atención oportuna y de calidad a las personas que sufren afecciones que requieren solución impostergable.

4. Asegurar que la organización, funcionamiento e infraestructura de los establecimientos sanitarios resguarden la dignidad de las personas y su privacidad, dispongan de tecnología de uso clínico habitual, preserven condiciones laborales adecuadas de su personal y fomenten el trato humanizado a los pacientes, por parte de todos los miembros del equipo de salud. Incrementar progresivamente la inversión en salud, de modo que permita enfrentar el costo real de la atención sanitaria y satisfacer, oportunamente y con calidad, las necesidades de salud de las personas.

5. Adoptar políticas públicas destinadas a disminuir el costo de los medicamentos, haciéndolos más accesibles a la población e impidiendo prácticas comerciales abusivas, que dañan a los pacientes. Adicionalmente, implementar un amplio listado de medicamentos genéricos con control de calidad y diversificando su oferta.

6. Fortalecer la investigación en salud, de acuerdo con las buenas prácticas clínicas, en aquellas materias prioritarias para el interés sanitario de la población, asegurando su financiamiento, la formación de investigadores de carrera e implementando un sistema técnico y autónomo que la apoye

7. Requerir la acreditación de las Escuelas de Medicina e instarlas a que, en la formación de los futuros médicos y profesionales de la salud, se ponga énfasis en el espíritu solidario y de servicio a la comunidad y en el comportamiento ético que exige el ejercicio de las profesiones de la salud.

Finalmente, las Academias Nacionales de Medicina de los países latinoamericanos reiteran su ofrecimiento para colaborar con los Gobiernos a fin de alcanzar los objetivos arriba señalados. 